\title{
Total fenestrated frozen elephant trunk technique for aortic repair of acute type $A$ aortic dissection
}

Homare Okamura, MD, PhD, Mamoru Arakawa, MD, PhD, Taro Takeuchi, MD, and

Hideo Adachi, MD, PhD, Tokyo, Japan

From the Department of Cardiovascular Surgery, Nerima Hikarigaoka Hospital, Tokyo, Japan.

Disclosures: Authors have nothing to disclose with regard to commercial support.

Received for publication July 31, 2018; revisions received Sept 4, 2018; accepted for publication Sept 8, 2018; available ahead of print Oct 22, 2018.

Address for reprints: Homare Okamura, MD, PhD, 2-11-1 Hikarigaoka, Nerima-Ku, Tokyo 179-0072, Japan (E-mail: homareo@jadecom.jp).

J Thorac Cardiovasc Surg 2019;157:e71-3

$0022-5223 / \$ 36.00$

Copyright () 2018 by The American Association for Thoracic Surgery

https://doi.org/10.1016/j.jtcvs.2018.09.022

- Video clip is available online.

In surgical repair for acute type A aortic dissection, extended aortic repair with a frozen elephant trunk (FET) reportedly reduces distal reintervention. ${ }^{1}$ Extended repair, however, has been shown to be associated with higher rates of early mortality than hemiarch repair. ${ }^{2}$ The complexity of

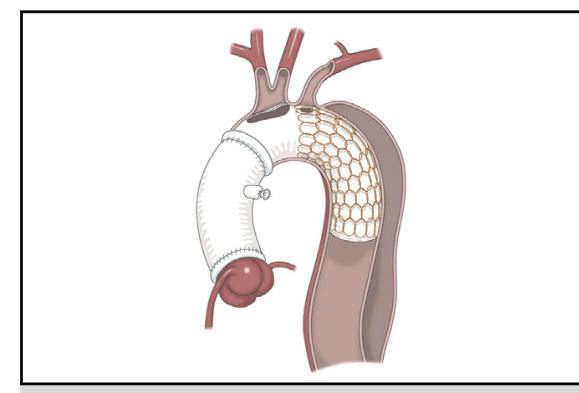

The total fenestrated frozen elephant trunk technique.

Central Message

The total fenestrated frozen elephant trunk technique is an effective procedure that accelerates aortic repair without the need for reconstructing the supra-aortic vessels.

See Commentary on page e75.

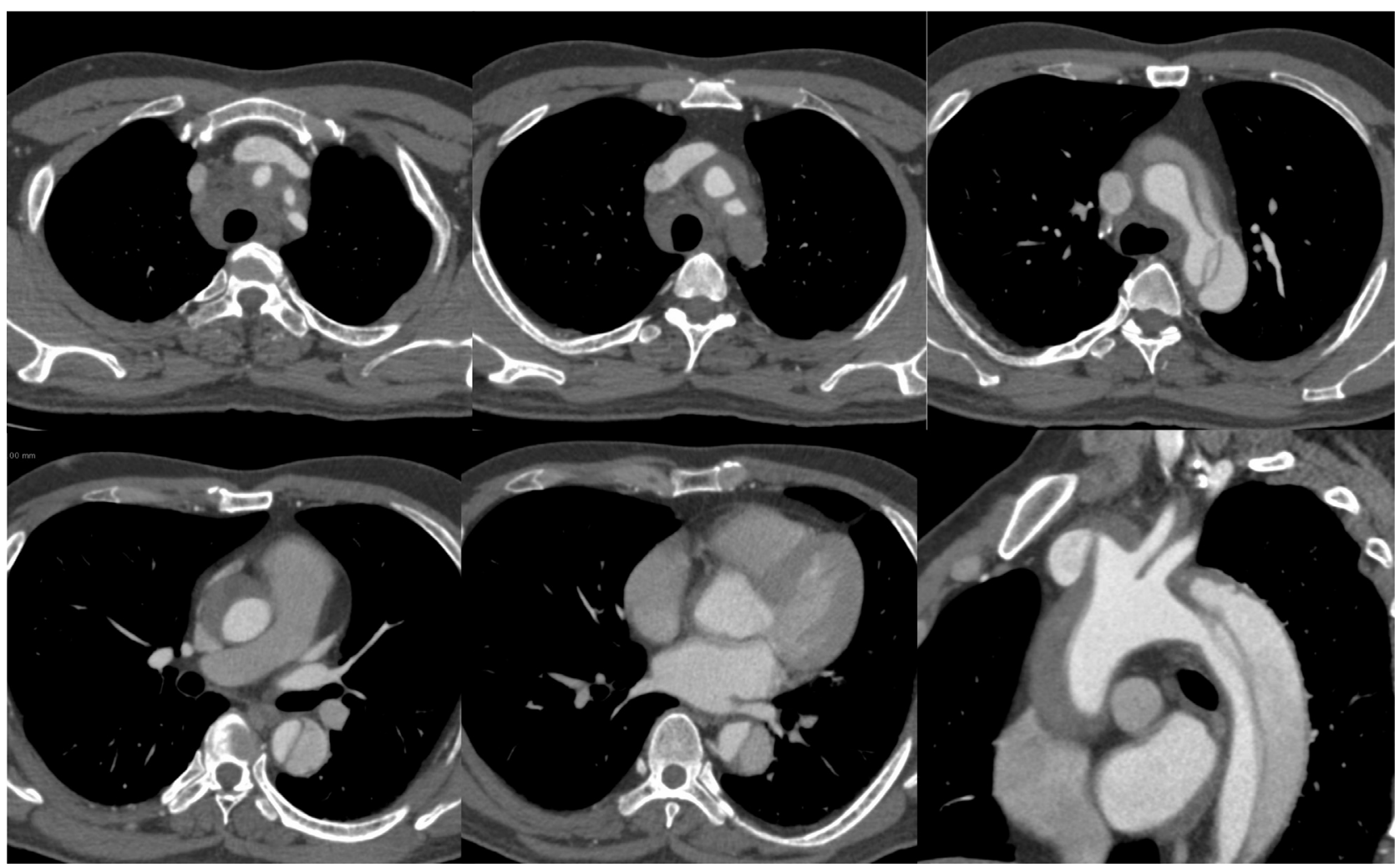

FIGURE 1. Preoperative contrast-enhanced computed tomographic scan. 

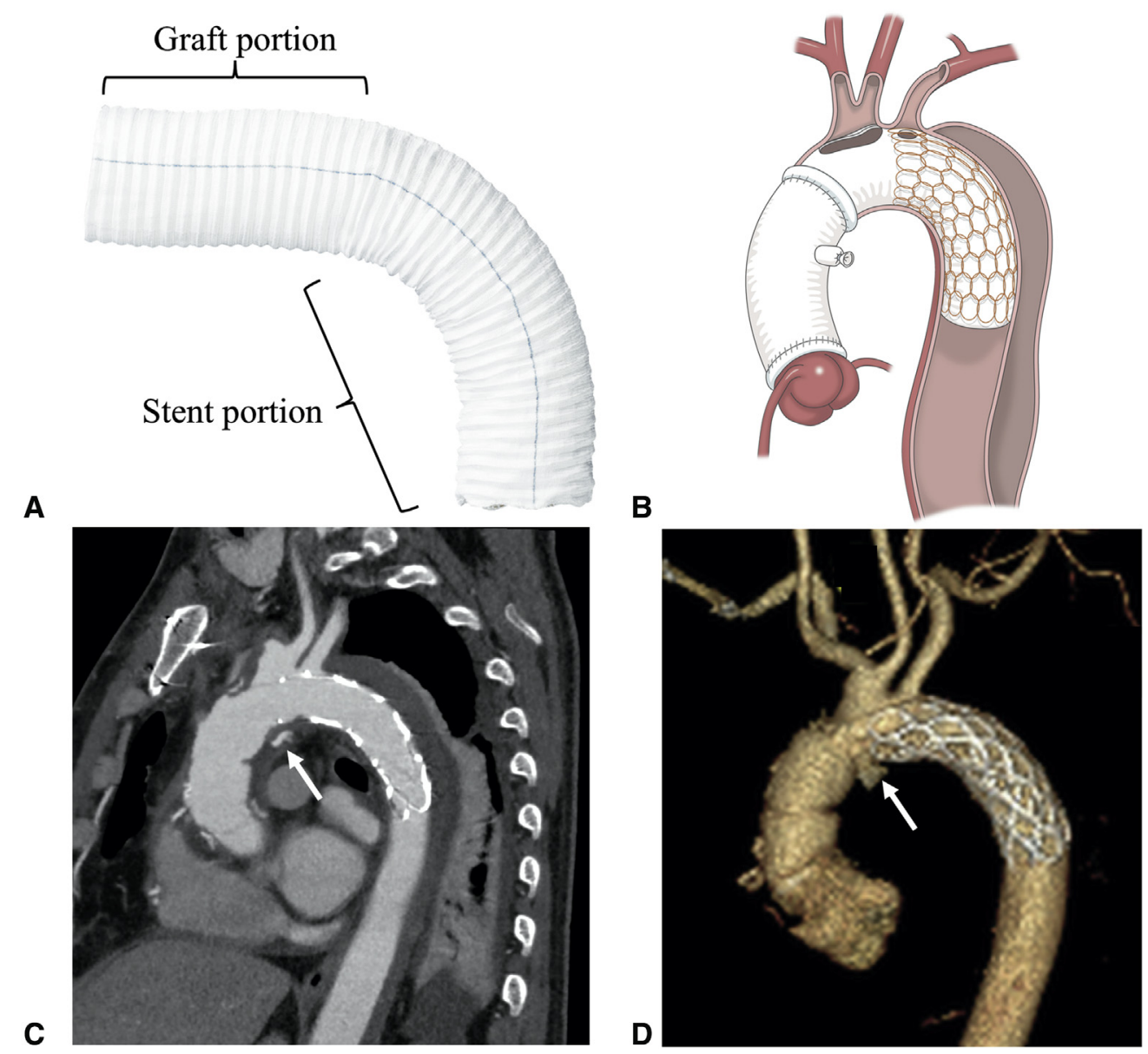

FIGURE 2. The total fenestrated frozen elephant trunk technique. A, Picture of the FROZENIX frozen elephant trunk graft (J-Graft FROZENIX; Japan Lifeline, Tokyo, Japan). B, Schema of the procedure. C, Sagittal view of the postoperative computed tomographic scan. D, Postoperative 3-dimensional computed tomography scan. Arrows indicate the distal anastomosis.

total arch replacement with the FET makes this procedure challenging in the acute setting. We report here a total fenestrated FET technique without the need for reconstructing the supra-aortic vessels.

\section{CLINICAL SUMMARY}

A 49-year-old man who presented with chest pain and paraplegia was transferred to our institution. His comorbidities included untreated hypertension. Contrast-enhanced computed tomography demonstrated acute type A aortic dissection and a bovine trunk. An intimal tear was found in the proximal descending aorta (Figure 1).

Emergency surgery was performed through a median sternotomy. Cardiopulmonary bypass was initiated through cannulation of the ascending aorta (with a modified Seldinger technique), the right atrium, and a left ventricular vent. When pharyngeal temperature decreased to $22^{\circ} \mathrm{C}$, hypothermic circulatory arrest was performed. Retrograde and antegrade cold blood cardioplegia were intermittently administered. Antegrade cerebral perfusion was not performed. The aorta was transected proximal to the innominate artery, and a commercially available FET graft (J Graft FROZENIX; Japan Lifeline, Tokyo, Japan) ${ }^{3}$ was manually curved and then deployed across the arch into the descending aorta. The J Graft FROZENIX comprises a stent portion and a graft portion (Figure 2, $A$ ). The diameter and length of the FET were $29 \mathrm{~mm}$ and $90 \mathrm{~mm}$, respectively. Subsequently, 2 fenestrations approximately 10 and $20 \mathrm{~mm}$ in size were manually created on the neck vessel sides of the stent and graft portions of the FET with scissors and electric cautery (Figure 2, $B$, and Video 1). After reinforcement with a felt strip, a 1-branched Dacron polyester fabric tube graft (J graft; Japan Lifeline) was anastomosed to the transected aorta with continuous 4-0 polypropylene sutures, as is commonly performed for the hemiarch repair. Adequate flow into the neck vessels was confirmed with blood pressure measurements in both arms and near-infrared spectroscopy of the head. A proximal anastomosis was performed with continuous 4-0 polypropylene sutures. The aortic crossclamp and total circulatory arrest times were 62 and 


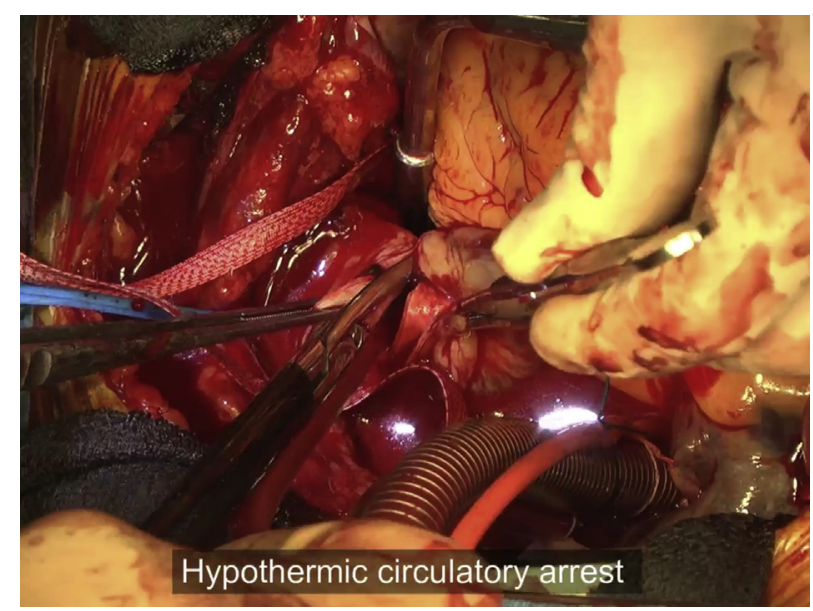

VIDEO 1. An intraoperative video that demonstrates the total fenestrated frozen elephant trunk technique with the FROZENIX graft (J-Graft FROZENIX; Japan Lifeline, Tokyo, Japan). Video available at: https:// www.jtcvs.org/article/S0022-5223(18)32523-6/fulltext.

23 minutes, respectively. The transfused blood contained $560 \mathrm{~mL}$ of red blood cell concentrates, $960 \mathrm{~mL}$ of freshfrozen plasma, and $250 \mathrm{~mL}$ of platelet concentrates.

The postoperative course was uneventful. The durations of stay in the intensive care unit and the hospital were 5 and 30 days, respectively. The patient's paraplegia resolved almost completely, with remaining motor dysfunction of the ankles. Postoperative computed tomography showed preserved blood flow into the supra-aortic vessels through the fenestrations in the FET and the thrombosed false lumen in the downstream aorta (Figure 2, $C$ and $D$ ). Follow-up computed tomography 3 months after surgery showed the thrombosed false lumen without endoleak.

Institutional review board approval was obtained for the fenestrated frozen elephant trunk technique. Consent from the patient was obtained for this case report.

\section{DISCUSSION}

In extended aortic repair of acute type A aortic dissection, it is ideal to choose a procedure that both can prevent late reintervention and is easy to perform, especially in critically ill patients. We previously reported a fenestrated FET technique in which left subclavian artery reconstruction is not necessary. ${ }^{4}$ Furthermore, in our total fenestrated FET technique, we aimed to seal off the intimal tear in the proximal descending aorta, preserve the blood flow to all the neck vessels, and ultimately shorten the aortic crossclamp and circulatory arrest times. The deployment of the FET across the arch and manual fenestration can be easily accomplished within 3 minutes. Given that the expected hypothermic circulatory arrest time is approximately $20 \mathrm{mi}-$ nutes, we have not routinely performed antegrade cerebral perfusion.

In our technique, the stent portion of the FROZENIX FET widens the inner cavity of the arch, allowing easy fenestration. The graft portion of the FET makes the distal anastomosis simple.

Patient selection for this technique is important. The total fenestrated FET technique should not be performed in cases of intimal tear in the arch, an enlarged aortic arch, and dissected neck vessels with reentry.

Zhou and colleagues ${ }^{5}$ previously reported another fenestrated FET technique. In their technique, the FET was fenestrated and mounted into a catheter before implantation. Because we fenestrated the FET under direct vision after deployment, precise positioning during deployment was not required, which can shorten the circulatory arrest time.

In conclusion, the total fenestrated FET technique for acute type A aortic dissection could be a simple and effective procedure in some patients. Long-term follow-up, however, is needed to confirm its effectiveness.

\section{References}

1. Dohle DS, Tsagakis K, Janosi RA, Benedik J, Kühl H, Penkova L, et al. Aortic remodeling in aortic dissection after frozen elephant trunk $\dagger$. Eur J Cardiothorac Surg. 2016;49:111-7.

2. Yan Y, Xu L, Zhang H, Xu ZY, Ding XY, Wang SW, et al. Proximal aortic repair versus extensive aortic repair in the treatment of acute type A aortic dissection: a meta-analysis. Eur J Cardiothorac Surg. 2016;49:1392-401.

3. Uchida N, Katayama A, Higashiue S, Shiono M, Hata M, Minami K, et al. A new device as an open stent graft for extended aortic repair: a multicenter early experience in Japan. Eur J Cardiothorac Surg. 2016;49:1270-8.

4. Okamura H, Arakawa M, Takeuchi T, Adachi H. The fenestrated frozen elephan trunk technique for acute type A aortic dissection. J Thorac Cardiovasc Surg. 2018;156:e75-7.

5. Zhou Q, Xue Y, Cao H, Pan J, Wang Q, Fan F, et al. Novel arch fenestrated sten graft for acute Stanford Type A aortic dissection with open antegrade implantation. Interact Cardiovasc Thorac Surg. 2018;26:369-75. 